\section{Tuberous sclerosis complex and polycystic kidney disease together: An exception to the contiguous gene syndrome}

\section{To the Editor:}

Tuberous sclerosis complex (TSC) is an autosomal dominant condition characterized by the presence of multiple, typically benign, tumors throughout various organ systems in the body. The organ systems most commonly affected are the brain, skin, heart, lung, and kidney. In addition, individuals with TSC may have facial angiofibromas, hypopigmented macules, shagreen patches (connective tissue nevi), seizures, and mental retardation. The incidence of TSC is between 1 in 6,000 and 1 in 11,000 births. ${ }^{1,2}$ The penetrance of the TSC1 and TSC2 genes is approximately $95 \%$, although the presentation of TSC varies widely. ${ }^{2,3}$

Mutations in two tumor suppressor genes, TSC1 and TSC2, have been shown to cause TSC. The TSC1 gene is located on chromosome $9 \mathrm{q} 34$ and codes for the protein hamartin, whereas the TSC2 gene is located on chromosome 16p13 and codes for the protein tuberin. Hamartin and tuberin come together to form a complex that inhibits several cell signaling pathways, namely, the $\mathrm{PI} 3 \mathrm{~K} / \mathrm{PKB}$ pathway (a growth and translation regulatory pathway), the glycogen synthase kinase $3 / \beta$-catenin/focal adhesion kinase/Ras-related homolog pathway (which involves cell adhesion, migration, and protein transportation), and the mitogen-activated protein kinase pathway, which is involved in cell growth and proliferation. ${ }^{4}$

Approximately two-thirds of TSC cases are caused by a de novo mutation, and the remaining are familial. ${ }^{1,4}$ Among the familial cases of TSC, mutations in the TSC1 and TSC2 genes are found with approximately the same frequency, whereas in sporadic cases of TSC, mutations in TSC2 are much more common, $80 \%$ versus $20 \%$, respectively. Most recent studies have found that individuals with TSC1 mutations typically have less severe disease than those with TSC2 mutations. ${ }^{1,2,5}$

In addition to the typical renal manifestations of TSC, such as angiomyolipomas, cysts, and oncocytomas, early-onset autosomal dominant polycystic kidney disease (ADPKD) has been observed in a number of individuals with TSC. ${ }^{5}$ Patients with TSC who have ADPKD are known to have a contiguous gene syndrome involving deletion of all or part of the TSC2 and PKD1 genes. ${ }^{6,7} \mathrm{ADPKD}$ is a systemic illness resulting in hyper- tension, hematuria, urinary tract infections, renal failure, and increased risk of cerebral aneurysms. Mutations of two genes are known to cause ADPKD; these are PKD1, which is located on chromosome 16p13.3 and codes for the protein polycystin 1 , and PKD2, which is located on chromosome 4q21-q23 and codes for the protein polycystin $2 .^{8}$ Although mutations in both $P K D 1$ and $P K D 2$ cause ADPKD, individuals affected with $P K D 1$ mutations predominate ( $85 \%$ PKD1 vs. $15 \%$ PKD2) and have more severe disease, leading to earlier onset of renal failure in patients with $P K D 1$ mutations versus those with $P K D 2$ mutations. ${ }^{9}$

The TSC2 and PKD1 genes are located next to one another in a tail-to-tail fashion on chromosome 16p13.3. Consequently, a large deletion involving the $3^{\prime}$ end of either gene often affects both the TSC2 and PKD1 and results in a contiguous gene syndrome. ${ }^{1,5-7}$ The TSC2/PKD1 contiguous gene syndrome typically manifests in infancy as severe ADPKD. In addition, loss of TSC2 gene activity may increase the pathogenicity of ADPKD, because tuberin loss affects proper polycystin- 1 localization. ${ }^{10}$

We present an unusual case in which a family presented with both ADPKD and TSC, and surprisingly had neither TSC2 nor PKD1 gene mutations.

In 1975 the proband (III-5) (Fig. 1) presented at the age of 8 years with a shagreen patch, toenail fibroma, and facial angiofibromas. The proband's father (II-2) also exhibited signs of TSC, specifically, facial angiofibromas, ungual and subungual fibromas, several shagreen patches and hypopigmented macules, and an achromic patch of the retinal pigmented epithelium in his left eye. The father had not been previously diagnosed with TSC. He did, however, have a previous diagnosis of polycystic kidney disease that had led to renal failure requiring dialysis 4 years prior at 51 years of age. After his TSC findings were discovered, the proband's father was diagnosed with TSC;

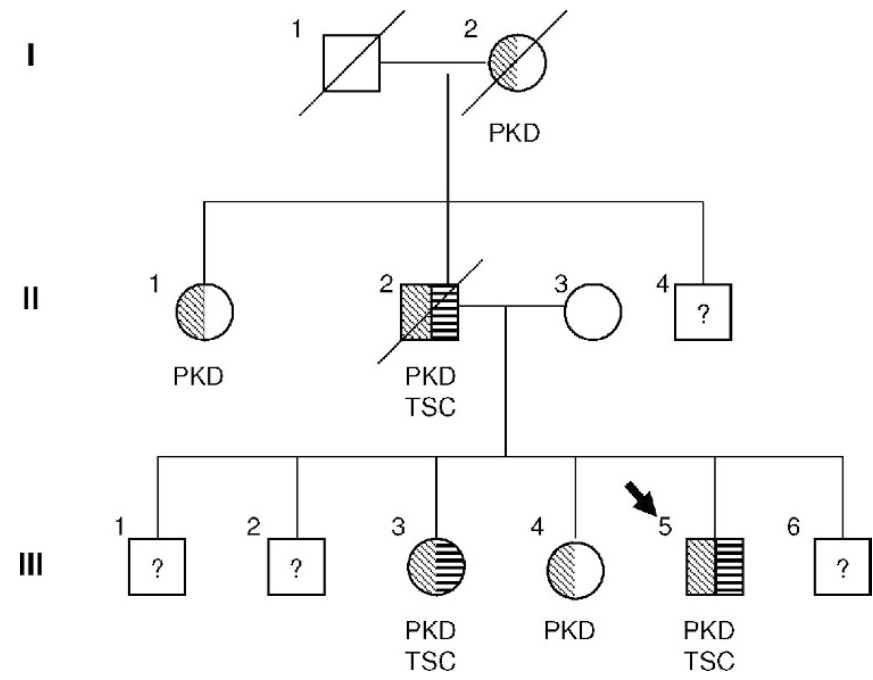

Fig. 1. Three-generation pedigree of the family indicating individuals affected with autosomal dominant polycystic kidney disease (ADPKD) as a result of a novel missense mutation $(\mathrm{R} 322 \mathrm{Q})$ of the PKD2 gene, as well as those affected with tuberous sclerosis complex (TSC) as a result of a four base-pair deletion (AACA at 2509-2512) of the TSC1 gene. Individual III-5 is the proband (arrow) originally enrolled in our TSC study. Individuals with ? were not available for analyses. 
his polycystic kidneys were thought to be a manifestation of his TSC. The proband's mother (II-3) and five siblings had no signs of TSC by our physical examinations, which included Wood's light.

The family was contacted 10 years later regarding participation in a TSC linkage study. The proband, his mother, his two older sisters (III-3 and II-4), and a paternal aunt (II-1) participated in the study. The proband's father recently died of cardiac problems, and the remaining siblings did not participate in the study.

All participants underwent physical and retinal examinations, nonenhanced computed tomography of the brain, renal ultrasound, and electrocardiogram. Significant findings were observed in both sisters. The older sister had TSC with multiple bilateral renal cysts and several calcified nodules in the lateral ventricles of the brain. Multiple hepatic and bilateral renal cysts in the younger sister led to a diagnosis of polycystic kidney disease. The paternal aunt was found to have multiple bilateral renal cysts, but no additional findings suggestive of TSC. No significant findings were observed in the proband's mother.

Lymphoblast DNA was obtained from the proband, both sisters, and the proband's mother and paternal aunt. Linkage studies were performed using genetic markers on 16p13.3 (D16S525, TSC2 $5202 \mathrm{~T}>\mathrm{C}$, KG8-located in the $3^{\prime}$ untranslated region of PKD1, D16S291, and SM7) and 9q34.3(GSN, ABL, ASS, and D9S65, which are GT repeat polymorphisms). ${ }^{4,11}$ The results of those studies suggested that the TSC phenotypes in the proband and older sister segregated with the same inferred genotypes of the markers for both chromosomes 16p13.3 and $9 \mathrm{q} 34.3$. We were able to determine that the proband and older sister inherited the same copy of chromosome 16p13.3 from their father, whereas the younger sister received the other copy of the father's chromosome 16p13.3. Direct sequencing of all coding exons of the TSC1 and TSC2 genes revealed the TSC disease-causing mutation to be on TSC1 exon 20, and consisted of a four base-pair deletion of AACA at 2509-2512. This mutation was only present in the proband and older sister.

Additional testing revealed a novel missense mutation (R322Q) of the PKD2 gene on chromosome 4q21-q23 present in the proband, both sisters, and the paternal aunt. The majority of PKD2 mutations described to date are nonsense mutations or frameshift mutations leading to premature stops. ${ }^{9,12}$ However, another disease-causing missense mutation at the same site as that observed in the family presented here (R322W) has been reported among PKD2-linked families of both Czech and Chinese ethnicities. ${ }^{12,13}$ In this mutation, the arginine is replaced with tryptophan, whereas in the case presented, the arginine is replaced with glutamine. This arginine at codon 322 of the PKD2 gene on chromosome 4q21-23 is remarkably conserved across species, indicating its importance this position. Indeed, as Reiterova et al. point out, this mutation occurs in a large extracellular loop of polycystin 2, and is likely to be necessary for proper folding of the protein, as well as interaction with other molecules. ${ }^{12}$

In summary, mutations in two genes, PKD1 on chromosome 16p13.3 and the less commonly seen mutation of PKD2 on chromosome 4q21-23, are known to cause ADPKD. In addition, mutations in two genes, TSC1 on chromosome 9q34 and TSC2 on 16p13.3, cause TSC. All reported cases of ADPKD coexisting with TSC have been the result of a contiguous gene syndrome in which both PKD1 and TSC2 (located very close to each other on chromosome 16p13.3) have been deleted, causing severe, early-onset ADPKD in addition to TSC. We have presented an unusual case of a family with both ADPKD and TSC in which neither of the disease-causing mutations is on chromosome 16p13.3. This family provides precedent for the need to screen for subtle mutations on PKD1 and PKD2 as well as TSC1 and TSC2 for patients who present with less severe TSC phenotypes and late-onset ADPKD symptoms.

Audrey C. Woerner, BA

Kit-Sing $A u, P h D$

Aimee T. Williams, MS

The University of Texas Medical School at Houston

Houston, Texas

Peter C. Harris, PhD

Biochemistry/Molecular Biology and Medicine

Mayo Clinic

Rochester, Minnesota

Hope Northrup, MD

Director, Division of Medical Genetics

The University of Texas Medical School at Houston

Houston, Texas

\section{References}

1. Sancak O, Nellist M, Goedbloed M, Elfferich P, et al. Mutational analysis of the TSC1 and TSC2 genes in a diagnostic setting: Genotype-phenotype correlations and comparison of diagnostic DNA techniques in tuberous sclerosis complex. Eur J Hum Genet 2005;13(6):731-741.

2. Lendvay TS, Marshall FF. The tuberous sclerosis complex and its highly variable manifestations. J Urol 2003;169(5):1635-1642.

3. Northrup H, Wheless JW, Bertin TK, Lewis RA. Variability of expression in tuberous sclerosis. J Med Genet 1993;30(1):41-43.

4. Au KS, Williams AT, Gambello MJ, Northrup H. Molecular genetic basis of tuberous sclerosis complex: from bench to bedside. J Child Neurol 2004;19(9):699-709.

5. Lewis JC, Thomas HV, Murphy KC, Sampson JR. Genotype and psychological phenotype in tuberous sclerosis. J Med Genet 2004;41(3):203-207.

6. Brook-Carter PT, Peral B, Ward CJ, Thompson P, et al. Deletion of the TSC2 and $\mathrm{PKD} 1$ genes associated with severe infantile polycystic kidney disease- a contiguous gene syndrome. Nat Genet 1994;8(4):328-332.

7. Sampson JR, Maheshwar MM, Aspinwall R, Thompson P, et al. Renal cystic disease in tuberous sclerosis: the role of the polycystic kidney disease 1 gene. Am J Hum Genet 1997;61:843-851.

8. Peters DJM, Spruit L, Saris JJ, Ravine D, et al. Chromosome 4 localization of a second gene for autosomal dominant polycystic kidney disease. Nat Genet 1993;5: 359-362.

9. Ong AC, Harris PC. Molecular pathogenesis of ADPKD: the polycystin complex gets complex. Kidney Int 2005;67(4):1234-1247.

10. Kleymenova E, Ibraghimov-Beskrovnaya O, Kugoh H, Everitt J, et al. Tuberindependent membrane localization of polycystin-1: a functional link between polycystic kidney disease and the TSC2 tumor suppressor gene. Mol Cell 2001;7(4):823832.

11. Northrup H, Kwiatkowski DJ, Roach ES, Dobyns WB, et al. Evidence for genetic heterogeneity in tuberous sclerosis: one locus on chromosome 9 and at least one locus elsewhere. Am J Hum Genet 1992;51(4):709-720.

12. Reiterova J, Stekrova J, Peters DJ, Kapras J, et al. Four novel mutations of the PKD2 gene in Czech families with autosomal dominant polycystic kidney disease. Hum Mutat 2002;19(5):573.

13. Zhang DY, Sun TM, Zhang SZ, Tang B, et al. Mutation detection of PKD2 gene in Chinese by denaturing high-performance liquid chromatograph. Chung Hua Hsueh Chuan Hsueh Tsa Chih 2004;21(3):211-214. 